\title{
LÓGICA BORROSA Y DECISIONES JUDICIALES: EL PELIGRO DE UNA FALACIA RACIONALISTA***
}

\section{Introducción: El planteamiento de un dilema}

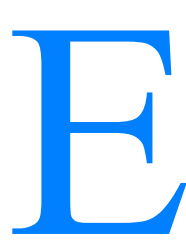

1 objeto de este artículo es un dilema que la lógica borrosa ${ }^{1}$ y la teoría de conjuntos borrosos pueden plantear en el análisis de las decisiones judiciales. De una forma resumida los dos cuerpos del dilema pueden formularse así:

judiciales puesto que suministra instrumentos lógicos para superar la crítica dirigida a la teoría lógico-deductiva de las decisiones judiciales.

\footnotetext{
* Deseo agradecer a E. Bulygin, P Comanducci, A. G. Conte, G. De Nova, R. Guastini y M. Taruffo sus estimulantes comentarios y observaciones a una primera versión de este artículo. Esta investigación ha sido llevada a cabo con el soporte financiero del Ministero dell'Universita e della Ricerca Scientifica e Tecnologica italiano.

** Traducción castellana de José Juan Moreso del original, Tecla Mazzarese: Fuzzy Logic and Judicial Decision-Making: The Peril of a Rationalist Fallacy. Es el texto de la ponencia presentada por la autora en el Congreso de Filosofia del Derecho en Homenaje al Prof. Ernesto Garzón Valdés, celebrado en Vaquerías (Córdoba-Argentina) en septiembre de 1992.

${ }^{1}$ Las expresiones inglesas «fuzzy logic», «fuzzy set theory» $\mathrm{y}$ «fuzziness» cuentan en castellano con dos adjetivos para su traducción, «borroso» y «difuso» («lógica borrosa» y «lógica difusa», «teoría de conjuntos borrosos» y «teoría de conjuntos difusos»). El sustantivo «fuzziness» es más difícil de traducir, pero la expresión «borrosidad» ha sido usada en algunas ocasiones. (vd. Voz «borroso» en José Ferrater Mora, Diccionario de Filosofia, 1, Barcelona: Círculo de Lectores, 1991, Alfredo Deaño, Introducción a la lógica formal, Madrid: Alianza, 1980, pp. 311-312 y, en el ámbito del análisis del razonamiento jurídico, Manuel Atienza Rodriguez, Sobre la analogía en el Derecho, Madrid: Civitas, 1986, pp.155-176. A. Deaño prefería traducir «fuzzy logic» por «lógica de los enunciados vagos» y hacía una pertinente consideración «No parece oportuno adoptar una traducción literal y hablar de "lógica vaga o borrosa". Lo vago no es la teoría sino el objeto que estudia»). Por esa razón, usaré el adjetivo «borroso» y sus derivados, «lo borroso», «borrosidad». En relación a la observación de Deaño, confiaré en que la expresión «lógica borrosa» no llama a nadie a engaño, tal y como sucede con «fuzzy logic», y que sea entendida de forma análoga a la expresión «teoría de conjuntos borrosos», en donde sí es claro que el carácter de borroso afecta al objeto y no a la teoría. [NOTA DEL TRADUCTOR].
} 
(b) La lógica borrosa no permite una reconstrucción racional de las decisiones judiciales puesto que -en virtud de sus rasgos peculiares- confirma más bien que supera la crítica dirigida a la teoría lógico-deductiva de las decisiones judiciales.

La tesis que me siento inclinada a vindicar es aquella expresada por el segundo cuerno del dilema. En mi opinión, el núcleo común de los diversos cálculos borrosos desarrollados desde $1965^{2}$ nos capacita para explicar la mayoría de dificultades teóricas con las que se encuentra la teoría lógico-deductiva de las decisiones judiciales, sin embargo estos cálculos no nos capacitan para resolver las dificultades mismas. Además, estoy convencida de que la tesis opuesta -aquella expresada por el primer cuerno del dilema- da lugar a lo que puede denominarse una forma de «falacia racionalista». Seguramente, la ocurrencia del término «lógica» en la frase «lógica borrosa» no puede tomarse como una garantía para el tipo de racionalidad que el valor jurídico de certeza pretende lograr.

Hasta ahora, la forma en que he planteado el tema de este artículo y la forma en que he establecido la tesis que argumentaré puede parecer críptica y esotérica. Ello no es sólo porque la referencia a la lógica borrosa seguramente no ha adquirido todavía un papel destacado en las investigaciones en teoría del derecho ni en los estudios sobre el razonamiento jurídico, sino por otras dos razones. En primer lugar, puede no ser bastante claro cuál es el trasfondo teórico de toda la cuestión. $\mathrm{Y}$ en segundo lugar, el dilema cuya formulación he sugerido puede contemplarse como aparente: como un problema más ficticio que real. Por estas dos razones se precisan algunas otras observaciones preliminares y explicativas.

\subsection{El trasfondo teórico jurídico del dilema: racionalismo normativo vs. irracionalismo normativo}

Según un punto de vista ampliamente extendido, las decisiones judiciales pueden ser como cuestión de hecho, y como cuestión de principio deben ser, de naturaleza lógico-deductiva, i.e. las decisiones judiciales pueden y deben tomarse siguiendo las reglas de inferencia de la lógica clásica. De este modo, es común caracterizar una decisión judicial como un silogismo -o en los análisis más recientes y refinados como una cadena de silogismos-

${ }^{2}$ L. A. Zadeh [1965] es el trabajo reconocido como el punto de partida oficial de la formalización lógica de lo borroso. Tal y como ocurre usualmente con la mayoría de nuevas ramas de la lógica formal, se han señalado varios antecedentes al punto de partida oficialmente reconocido, relacionados con los cálculos borrosos, principalmente cuando «borrosidad» $\mathrm{y}$ «vaguedad» se conciben como sinónimos, o al menos referidos a nociones relacionadas. Sobre los antecedentes de los cálculos borrosos. cf. e.g. S. Haack [1974, p. 112] y H. Faas [1991, cap. $1]$. 
que se denomina «judicial» no en virtud de alguna característica lógica peculiar, sino simplemente en virtud de producirse en el ámbito de la actividad judicial. ${ }^{3}$

A pesar de la variedad de deficiencias y de aspectos insatisfactorios que algunos estudiosos han achacado a tal punto de vista, la teoría del silogismo judicial disfruta todavía de una aceptación amplia puesto que, más allá de toda crítica, es aún concebida como la principal garantía de la racionalidad de las decisiones judiciales, y como un medio necesario para asegurar que el tan apreciado valor de certeza jurídica pueda ser logrado.

De esta forma, no es sorprendente que cualquiera que se atreva a plantear los problemas con que se enfrenta la teoría lógico-deductiva de las decisiones judiciales sea inmediatamente acusado como irracionalista y condenado como un defensor del decisionismo y del subjetivismo arbitrario del poder judicial. No es casual -por mencionar solamente un ejemplo muy conocidoque el último punto de vista sobre derecho y lógica de $\mathrm{H}$. Kelsen haya sido catalogado como una expresión del irracionalismo normativo ${ }^{4}$. No es tampoco casual que el debate iniciado por este último punto de vista kelseniano se haya centrado en la oposición entre racionalismo normativo e irracionalismo normativo.

Contra este trasfondo, la lógica borrosa podría adquirir un papel relevante, mostrando una vía de salida a esta estéril y engañosa oposición. Dejando de lado, sólo por el momento, que existen elementos cuyo comportamiento lógico es el objeto de la lógica borrosa, lo que merece la pena remarcar ahora es que tales elementos pertenecen al conjunto de cuestiones cuya significación es la piedra angular de la disputa entre los llamados racionalistas normativos e irracionalistas normativos: tal significación es disminuida o directamente ignorada por los primeros $\mathrm{y}$, por el contrario, es puesta de manifiesto y subrayada por los últimos.

\subsection{Las tesis contrapuestas del dilema y su relación con la pretensión de racionalidad de las decisiones judiciales}

La importancia que la lógica borrosa puede adquirir al analizar las decisiones judiciales es incuestionable, aunque está abierto el papel específico que la lógica borrosa puede jugar (o puede asumirse que jugará) en tal empresa. Por otra parte -como ya he argumentado- la lógica borrosa puede usarse como un medio para librarse de la oposición racionalismo normativo/irracionalismo

\footnotetext{
${ }^{3}$ Cf. e.g, G. Kalinowski [1965, p. 174]: «Pero existen razonamientos normativos, mal que les pese a los neopositivistas. Y cuando se utilizan en el curso de la aplicación del derecho se les llama silogismos jurídicos».

4 Como es bien sabido el término «Normirrationalismus» fue sugerido por O. Weinberger [1981] en relación a la última concepción sobre derecho y lógica de Kelsen.
} 
normativo y también como un medio para fundamentar de una forma nueva la misma oposición. La posibilidad de estas evaluaciones de signo contrario muestra claramente que el dilema que plantea este artículo es real y no aparente. Los resultados obtenidos mediante cada una de las tesis del dilema difieren de manera radical.

En verdad, de acuerdo a la primera tesis del dilema, se da por garantizado que una reconstrucción lógica (muy a menudo simplemente en el sentido de formal) es una razón suficiente para asegurar que cualquiera que sea el objeto de la reconstrucción lógica, dicho objeto cumple los estándares de racionalidad. (Si esto es así -por sugerir un ejemplo- entonces el razonamiento del sin sentido devendría una instancia de razonamiento racional, en tanto que un cálculo lógico capturara y formalizara alguna -o incluso la mayoría- de sus modelos ${ }^{5}$.) Para este punto de vista ingenuo sobre el formalismo lógico he sugerido, al inicio de este artículo, el término «falacia racionalista».

Por el contrario, según la segunda tesis del dilema una reconstrucción lógica como tal no garantiza necesariamente todos los estándares de racionalidad del objeto con el que trabaja. Una reconstrucción lógica podría ser -como parece adecuado sostener en relación a la lógica borrosaun modelo explicativo (uno entre varios y diferentes posibles) de la materia que constituye su objeto.

\subsection{Partes principales de este artículo y sus contenidos}

Este artículo está dividido en dos partes principales. En la primera parte me ocuparé de los fundamentos según los cuales la lógica borrosa puede contemplarse como un instrumento conceptual adecuado para dar cuenta de algunos rasgos relevantes de las decisiones judiciales. En la segunda parte me centraré en el dilema que la lógica borrosa puede plantear al análisis de las decisiones judiciales.

\section{Borrosidad y decisión judicial}

Es claro que la lógica borrosa no ha adquirido todavía una papel prominente en las investigaciones de teoría del derecho y, en particular, está lejos de gozar de una atención amplia en las investigaciones sobre las decisiones judiciales. De esta forma, en esta parte de mi trabajo, en primer lugar (1.1.) adelantaré algunas observaciones que pueden explicar la razón por la cual

\footnotetext{
${ }^{5}$ El ejemplo no es completamente ficticio. Se ha elaborado realmente una lógica del sin sentido, e.g. por S. Halldén y por R. Routley. Para unas referencias bibliográficas completas y unos pocos comentarios sobre el tema, cf. e.g. S. Haack [1978, p. 163] y A. Urquhart [1986, p. 1091].
} 
el tratamiento formal de lo borroso ha sido largamente ignorado par los teóricos del derecho. Estas observaciones no comportan, obviamente, ni la justificación ni el acuerdo con tal actitud. Más bien, deben entenderse como un intento de señalar algunas asunciones en las cuales descansa la engañosa oposición entre racionalismo e irracionalismo normativo.

A continuación (1.2.), trataré de esbozar una caracterización de la noción de «borrosidad», señalando algunas dificultades a las que tal noción está ligada.

Finalmente (1.3.), volveré a un examen más detallado del papel que la lógica borrosa puede adquirir en el tratamiento de la decisión judicial, mencionaré tres tipos principales de crítica dirigida a la teoría lógico-deductiva de las decisiones judiciales y me centraré en el papel potencial de la lógica borrosa para el análisis de las decisiones judiciales en virtud a la vez de la borrosidad del lenguaje judicial (1.3.1.) y de la borrosidad (o más correctamente: de la naturaleza aproximada) del razonamiento judicial (1.3.2.).

\subsection{Lógica borrosa: Un aparato conceptual marginado en las investigaciones de teoría del derecho}

Hasta donde conozco, muy pocos teóricos del derecho han prestado atención, en las varias cuestiones de las que se ocupan, a la importancia de los recientes y cada vez más abundantes desarrollos del tratamiento lógico formal de lo borroso.

Podría ser que la falta de interés fuera aparente, que no fuese el resultado de una reacción querida, sino un fenómeno de naturaleza contingente: la lógica borrosa y la teoría de conjuntos borrosos son campos de investigación recién nacidos, tratados por un número relativamente limitado de adeptos que usan instrumentos lógicos y matemáticos muy sofisticados. Aunque esta hipótesis es sólida, no es completamente convincente. Es claro que en los años cincuenta y sesenta la recién nacida lógica deóntica moderna dio lugar a expectativas muy confiadas (a veces incluso demasiado confiadas) en su potencial heurístico para investigaciones jurídicas tanto tradicionales como pioneras.

Así, es muy probable que la falta de interés en el tratamiento formal de lo borroso sea real, que sea el resultado de una reacción querida de rechazo desde una resuelta concepción, de naturaleza teórica: según una opinión profundamente enraizada, los teóricos del derecho no hacen ningún esfuerzo ni están interesados por los instrumentos conceptuales que les habiliten para elaborar, ocuparse y tomar en cuenta debidamente la borrosidad. Más bien, buscan medios que les permitan librarse de ella y cuando tal empresa no puede ser lograda con éxito completo, hacen lo posible para limitar y reglar todo aquello que -en cualquier contexto jurídico- sea borroso o pueda convenirle alguna especie de borrosidad. 
Los dos argumentos que soportan esta segunda hipótesis son suministrados por: (i) una explicación plausible de la hipótesis que en este punto puede sugerirse en relación con las reacciones radicalmente diferentes hacia los cálculos deónticos y los cálculos borrosos respectivamente y (ii) la actitud mayoritaria entre los lógicos hacia el tratamiento formal de la borrosidad.

(i) Los cálculos borrosos - contrariamente a lo que ocurre con la mayoría de cálculos deónticos- no son una extensión de la lógica clásica, pertenecen a lo que normalmente se denomina «lógicas divergentes» ${ }^{6}$, esto es, a aquellas lógicas en las cuales algunas leyes fundamentales de la lógica clásica no se mantienen (i.e.: no son válidas). A la vista de ello, las respuestas distintas de los teóricos del derecho a estas dos nuevas ramas de la lógica formal moderna pueden obtener una explicación muy simple.

En lo que a la lógica deóntica concierne, varios teóricos del derecho se han sentido confiados, desde el principio mismo de sus primeros desarrollos, porque la mayoría de sus cálculos -que son una extensión de la lógica clásica- preservan los estándares de racionalidad que la teoría jurídica trata de lograr. En relación a ello es interesante recordar la forma en que G. H. von Wright describe la reacción de Kelsen en su primer contacto con la lógica deóntica en $1952^{7}$ :

[Kelsen] quedó muy impresionado acerca de las posibilidades que la lógica deóntica parecía abrir para la vindicación de algunos aspectos básicos de su propia teoría «pura» del derecho. En particular, buscaba fundamento en la lógica para su idea según la cual un orden jurídico es necesariamente cerrado, i.e. no hay «lagunas» en el derecho, y para la idea según la cual un orden jurídico debe estar libre de «contradicciones» ${ }^{8}$.

\footnotetext{
${ }^{6}$ Una clara caracterización de la noción de «lógica divergente»-que captura el núcleo de los problemas a los que esta noción da lugar- es formulada por W. V. Quine [1970, pp. 80-81]: «La clase de desviación que ahora hemos de considerar [...] no trata simplemente de cambiar de métodos de producción de la clase de las verdades lógicas, sino de cambiar esa clase misma. No se trata tampoco de un simple cambio de fronteras entre lo que se ha de llamar verdad lógica y lo que merece el nombre de verdad extra-lógica. Se trata, más bien, de recusar abiertamente por considerarla falsa una parte de nuestra lógica» (subrayado mío). *El análisis de Quine de la lógica divergente en las pp. 80-94 no menciona las cuestiones específicas que afectan al tratamiento de la borrosidad o de la vaguedad -borrosidad y vaguedad son a menudo concebidas como nociones equivalentes-. La lógica borrosa y la teoría de conjuntos borrosos son señalados explícitamente como un caso paradigmático de lógicas divergentes con una cierta extensión en S. Haack [1978, pp. XII, 35, 162-169].

* La traducción del texto de Quine se ha obtenido de la traducción castellana de Manuel Sacristán (Filosofia de la lógica, Barcelona: Alianza, 1973, p. 140), la expresión «lógicas divergentes» como traducción de «deviant logics» también es de Manuel Sacristán. [NOTA DEL TRADUCTOR]

${ }^{7}$ Debe advertirse que von Wright rememora la visita de Kelsen -la única- a Finlandia, y que esta ocurrió precisamente un año después de la publicación de dos de sus artículos, a saber G. H. von Wright [1951a] y [1951b], que son usualmente reconocidos como el punto de partida oficial en el campo de la moderna lógica deóntica.

${ }^{8}$ G. H. von Wright $[1985,269]$.
} 
En lo que concierne a la lógica borrosa, varios teóricos del derecho podrían sentirse más bien remisos que confiados hacia ella puesto que -siendo la lógica borrosa una lógica divergentees dudoso qué tipo de racionalidad (si alguna) puede convenir a sus cálculos. Además, en virtud de su objeto específico la lógica borrosa tiende a adscribir importancia a aquello de lo que los teóricos del derecho tratan de liberarse en cualquier contexto jurídico.

(ii) En lógica -de forma similar a como ocurre en teoría jurídica- la borrosidad es concebida principalmente como una fuente de problemas y dificultades. Así, de hecho existe una especie de escepticismo hacia los intentos de su formalización. Los argumentos en los que está basado tal escepticismo no difieren de forma significativa de las razones ya avanzadas para la eliminación de la borrosidad de los contornos jurídicos.

Aunque se reconoce que la borrosidad es un rasgo peculiar de los lenguajes naturales, varios lógicos destacados consideran aún incómodo tenerla en cuenta en sus cálculos formales. En palabras de S. Haack:

una razón importante para construir sistemas formales de lógica consiste en facilitar cánones precisos de validez, una ventaja importante de la lógica formal sobre la argumentación informal no reglada es su mayor rigor y exactitud. Desde este punto de vista no es sorprendente que Frege y Russell hayan contemplado la vaguedad como un defecto de los lenguajes naturales, que debe ser eliminado de un lenguaje formal aceptable. [...] Tal vez esto sugiere que sería apropiado simplemente excluir los enunciados vagos como implausibles candidatos para el tratamiento lógico. ${ }^{9}$

Merece la pena observar que en lo referente al tema en cuestión, «vaguedad» es a menudo usado como sinónimo de «borrosidad» y cuando no es así la vaguedad es concebida sin duda como un ejemplo paradigmático de borrosidad o como una de sus principales fuentes.

\subsection{Una caracterización tentativa de «borrosidad»}

La observación terminológica realizada al final del último párrafo presupone una cuestión aún no resuelta, i.e. definir la noción de «borrosidad». La tarea no es nada sencilla. Por el contrario, tal y como se sostiene que la noción de «vaguedad» es vaga ${ }^{10}$, en el mismo sentido la noción de «borrosidad» es borrosa. Es así al menos por cuatro razones: (a) la frontera entre los casos en que una proposición o bien un predicado puede decirse, sin duda, que sea borroso o no es en sí mismo borrosa ${ }^{11}$; (b) la borrosidad de una

\footnotetext{
${ }^{9}$ S. Haack [1978, pp. 162-163].

${ }^{10}$ Un punto de vista diferente es mantenido por H. Faas [1991, pp. 3-4].

${ }^{11}$ En relación a la vaguedad una observación similar se halla en C. Luzzati [1990, pp. 4]. En relación a la textura abierta, una observación similar se sigue del análisis de tal noción suministrado par F. Waismann [21951, pp 118-124] por lo que se refiere a la verificabilidad de los conceptos empíricos, y es explícitamente resaltado e.g. por R Guastini [1992 pp. 115-117] por lo que se refiere a la teoría de la interpretación jurídica.
} 
proposición igual que la de un predicado puede ser dependiente del contexto (i.e. la misma entidad puede ser comprendida como borrosa o no en relación a sus diferentes instancias); (c) parece difícil dibujar de una forma clara la estructura de relaciones y el complejo de semejanzas y diferencias que hay entre la noción de «borrosidad» y aquellas otras nociones cercanas como «vaguedad», «incertidumbre», «indeterminación», «genericidad» que seguramente pertenecen al mismo campo semántico ${ }^{12}$; (d) parece difícil definir el ámbito de los datos que podrían ser (o más bien que deberían ser) calificados como borrosos o el ámbito de los predicados a los que conviene (o puede convenir) la borrosidad, la cuestión es raramente explicitada y los ejemplos existentes en la literatura son más bien de naturaleza diferente ${ }^{13}$. A pesar de tales dificultades, al menos el núcleo de la noción de "borrosidad" puede captarse esbozando lo que puede tomarse como el objeto específico de los cálculos borrosos. En expresión de Haack:

En resumen, podría considerarse la lógica borrosa como el resultado de dos etapas de «borrosificación» [fuzzification]: el paso de una lógica bivalente a una lógica de innumerables valores como resultado de permitir grados de pertenencia a los conjuntos denotados por los predicados del lenguaje objeto y el paso a muchos valores de verdad borrosos y contables como un resultado de tratar el predicado metalingüístico «verdadero» como vago en sí mismo. ${ }^{14}$

En otras palabras, los cálculos borrosos se ocupan de proposiciones borrosas que -al contrario que las proposiciones susceptibles de verdad y falsedad de las que se ocupa la lógica clásica bivalente- pueden ser verdaderas o falsas hasta cierto grado según la escala de «valores veritativos borrosos»

12 Debe observarse de todas formas que la noción de «vaguedad» no se distingue, a menudo, de la noción de «borrosidad», y que se han llevado a cabo algunos intentos de distinguir vaguedad de otras nociones próximas. Ver e.g. la distinción entre vaguedad y textura abierta propuesta par E Waismann [21951, pp. 120-121], la distinción entre vaguedad e incertidumbre trazada por S. Haack [1974, pp 110-11], la distinción entre vaguedad, ambigüedad, genericidad y generalidad subrayada por C. Luzzati [1990, pp. 46-52], la distinción entre vaguedad y ambigüedad indicada por H. Faas [ 1991, cap. 1]. También puede verse las relaciones múltiples establecidas entre vaguedad, ambigüedad y los denominados conceptos contestados sugeridos por E. Diciotti [1992, pp. 98-113]. Aunque varias observaciones sobre la estructura compleja de relaciones que existen entre vaguedad y otras nociones cercanas puede ser contemplada como convincente, sin embargo es dudoso si las mismas observaciones pueden ser tomadas como verdaderas en relación a la borrosidad como tal. Es decir que es dudoso si «vaguedad» puede denotar la misma noción que «borrosidad». Realmente, parece adecuado mantener que la vaguedad es sólo una de las diferentes fuentes posibles de la borrosidad junto a la ambigüedad, la generalidad, la genericidad, y la incertidumbre.

${ }^{13}$ Tal dificultad se parece mucho a la señalada por S. Haack [1974, pp. 110] en relación a la vaguedad: «Los ejemplos discutidos en la literatura bajo el título «vaguedad» son de una variedad extraordinaria y no es fácil especificar qué tienen en común. [...] Un rasgo compartido por la mayoría de ejemplos es que el predicado en cuestión es tal que para algún(os) sujeto(s) existe incertidumbre sobre si el predicado se le(s) aplica. La incertidumbre de aplicación no es, sin embargo, coextensiva con la vaguedad».

${ }^{14}$ S. Haack [1978, pp. 66]. 
asumida en un cálculo determinado. Una proposición será borrosa si al menos contiene un predicado borroso. De manera semejante, un conjunto borroso -al contrario que en la teoría de conjuntos clásica- admite diferentes grados de pertenencia de las entidades denotadas por un predicado borroso determinado.

\subsection{El papel potencial de la lógica borrosa en el análisis de las decisiones judiciales}

La teoría tradicional lógico-deductiva de las decisiones judiciales no deja lugar para la borrosidad: simplemente se ignora cuando no directamente se niega, que la borrosidad sea un rasgo de las decisiones judiciales, tanto en relación a sus elementos constitutivos como en relación a los modelos de razonamiento que maneja el decisor para tomar una decisión judicial. Así, una decisión judicial es concebida como una inferencia lógica trazada según un esquema silogístico: la norma del caso (i.e. la norma expresada por la conclusión de una decisión judicial) se contempla como la conclusión lógica de un esquema silogístico donde la premisa mayor (también llamada premisa jurídica) es suministrada por la(s) norma(s) general(es) aplicable(s) y la premisa menor (también llamada premisa fáctica) es suministrada por la descripción del hecho (de los hechos) en cuestión.

Aunque tal aparato conceptual parece asegurar los estándares de racionalidad en las decisiones judiciales que la mayoría de teóricos del derecho nunca se atreverían a abandonar, aún la teoría tradicional del silogismo judicial ha sido el objeto de varias críticas principalmente centradas en tres cuestiones: (a) los problemas que resultan de la determinación de la cuestión de derecho no tienen un enfoque satisfactorio cuando se habla de la formulación de la premisa mayor o jurídica del silogismo judicial; (b) los problemas que resultan de la determinación de la cuestión de hecho no tienen un enfoque satisfactorio cuando se habla de la formulación de la premisa menor o fáctica del silogismo judicial, (c) la validez jurídica (que se aplica a las normas) no puede tener un comportamiento lógico (si tiene alguno) similar al comportamiento lógico de la verdad (que se aplica a las proposiciones de las que se ocupa la teoría tradicional del silogismo y la lógica clásica).

Ahora bien, parece adecuado sostener que la crítica dirigida a la teoría del silogismo judicial -centrada en las tres cuestiones antes mencionadas tiene sus raíces en la borrosidad múltiple de las decisiones judiciales (o para ser más precisa: en la borrosidad múltiple del lenguaje judicial) y ello sin referirse a las diferentes maneras en que tal crítica se ha expresado o basado. Realmente la crítica relacionada con las dos primeras cuestiones (i.e. la determinación de la cuestión de derecho y de la cuestión de hecho) surge, y puede ser básicamente tomada, en términos de borrosidad del lenguaje en el que la cuestión de derecho y la cuestión de hecho son respectivamente formuladas. 
A su vez la crítica relacionada con la tercera cuestión (i.e. el comportamiento lógico de la validez jurídica) puede ser explicada -aunque probablemente no del todo- considerando el razonamiento judicial bajo la perspectiva del razonamiento borroso, i.e. bajo la perspectiva del razonamiento aproximado cuyas pautas lógicas -sea lo que fuere aquello que lógico significa en tal perspectivason divergentes de las leyes de la lógica clásica.

\subsubsection{DECISIONES JUDICIALES Y BORROSIDAD DEL LENGUAJE JUDICIAL}

Por «lenguaje judicial» me refiero al conjunto de lenguajes diferentes y necesarios en las decisiones judiciales para formular (a) la cuestión de derecho (la llamada premisa mayor o jurídica de un silogismo judicial); (b) la cuestión de hecho (la llamada premisa menor o fáctica del silogismo judicial); (C) la norma del caso (la llamada conclusión del silogismo judicial); (d) la justificación de la decisión judicial misma, cuando la justificación es requerida por el derecho procesal como un elemento constitutivo de las decisiones judiciales.

El lenguaje judicial -así definido- es un complejo de enunciados pertenecientes a una de las cuatro diferentes clases de lenguaje jurídico siguientes: (i) lenguaje jurídico stricto sensu; (ii) lenguaje jurídico de la dogmática, (iii) lenguaje jurídico axiológico; (iv) lenguaje jurídico de la determinación de los hechos ${ }^{15}$. No es difícil mostrar que cada uno de estos lenguajes jurídicos puede ser tildado de borroso.

(i) Borrosidad del lenguaje jurídico stricto sensu. Por "lenguaje jurídico stricto sensu" me refiero al lenguaje en el que las normas de un orden jurídico dado están formuladas. En otras palabras: me refiero al conjunto de formulaciones normativas que expresan las normas cuya totalidad constituye un orden jurídico dado ${ }^{16}$. El lenguaje jurídico stricto sensu es el lenguaje en el que la cuestión de derecho y la norma del caso de una decisión judicial están formuladas.

Ahora bien, el lenguaje jurídico stricto sensu es borroso en un doble sentido. Para ser más precisa su borrosidad surge de dos fuentes diferentes: (a) la forma en que las formulaciones normativas están escritas, (b) la estructura compleja de las relaciones que hay (o puede asumirse que hay) entre las diferentes normas expresadas por las formulaciones normativas cuya totalidad constituye un orden jurídico dado ${ }^{17}$.

\footnotetext{
${ }^{15}$ Es innecesario decir que los términos de la distinción sugerida entre clases diferentes de lenguaje jurídico no son ni conjuntamente exhaustivos ni mutuamente excluyentes.

16 Este uso de «lenguaje jurídico» conlleva simplemente la naturaleza dependiente del lenguaje de las normas (jurídicas) y deja abierta la debatida cuestión acerca de la naturaleza lingüística o extralingüística de las normas. La tesis de la naturaleza dependiente del lenguaje de las normas (jurídicas) es explícitamente establecida por G. H. von Wright [1963, pp. 941].

${ }^{17}$ Las dos fuentes de borrosidad son señaladas por Wróblewski, que las denomina «borrosidad de las normas jurídicas» y «borrosidad de la validez» respectivamente. (Cf. Wróblewski, 1983, la misma distinción aparece en A. Peczenik/J. Wróblewski [1985]). La forma en que Wróblewski analiza la «borrosidad de los sistemas jurídicos» seguramente influye y condiciona mi propia concepción sobre el doble sentido de acuerdo al cual el lenguaje jurídico stricto sensu es borroso, sin embargo en lo que continua no seguiré tan de cerca el artículo de Wróblewski, tratando de desarrollar sus intuiciones por una vía de razonamiento parcialmente diferente y usando una terminología diversa. Distinciones similares a la que examine han sido trazadas en relación a nociones muy cercanas a la noción de borrosidad, a saber: textura abierta y variedad. La textura abierta de las normas jurídicas y la textura abierta del derecho son distinguidas y tratadas par H. Hart [1961, cap 7]; la vaguedad de las normas y la vaguedad de los ordenes jurídicos son distinguidos y tratados por C. Luzzati [1990].
} 
La primera fuente de borrosidad se debe a la forma en que las formulaciones normativas están escritas, i.e. los términos de una formulación normativa resultan normalmente en un enunciado que es borroso en virtud de razones semánticas y sintácticas ${ }^{18}$. Dejando aparte la clase de borrosidad debida a razones sintácticas -que el legislador podría prevenir casi en todas las ocasiones, prestando una más cuidadosa atención a las reglas gramaticales de su propio lenguajetomaré en cuenta la borrosidad de carácter semántico. Esta clase de vaguedad es realmente muy difícil de prevenir y de evitar. De hecho, los diversos términos que aparecen en las formulaciones normativas están lejos, aunque por razones diversas, de ser claramente definidos (o definibles) y de permitir al intérprete establecer cuál pueda ser el «significado exacto» de una formulación normativa. Esto no es únicamente en virtud del uso de e.g. términos del lenguaje común, de términos valorativos, de términos psicológicos, de términos jurídicos que pueden conllevar significados divergentes en razón de las diversas definiciones jurídicas que se les han dado. Es también en virtud del extendido empleo de los denominados estándares jurídicos o conceptos jurídicos indeterminados [en alemán, en inglés lo general, en italiano: clausole generali] y/o máximas de experiencia [en alemán: Erfahrunsgssatze, en inglés: common sense notions, en italiano: massime d'esperienza $]^{19}$ tanto como en virtud de la tendencia a no fijar claramente las sanciones jurídicas, dejando al que ha de tomar la decisión la tarea de su determinación en una clase dada, en el alcance de una

${ }^{18}$ Esta fuente doble de borrosidad del lenguaje jurídico legal es explícitamente señalada por J. Wróblewski [1983, p.322] al analizar lo que denomina «borrosidad de las normas jurídicas». Una observación similar se halla también en A. Peczenick/J. Wróblewski [1985, p.32]. Como es bien sabido tanto los problemas semánticos como sintácticos a los que pueden dar lugar los textos jurídicos son un tema clásico tratado en la interpretación jurídica, al menos desde el análisis de A. Ross [1958, pp.123-128, pp.134-135].

${ }^{19}$ Presta atención a esta clase de nociones, en la perspectiva del tratamiento lógico-formal de la borrosidad, L. Philipps [1990, p.823]. Sobre borrosidad y conceptos jurídicos indeterminados merece la pena citar a J. Wróblewski [1983, p.326] que afirma. «No es que el legislador, para la certeza del derecho y la eficacia en el encauzamiento de los comportamientos, evite la indeterminación de las reglas que formula, por el contrario, puede usar el lenguaje borroso de los conceptos jurídicos indeterminados para imponer el peso de la decisión sobre el que debe tomarla y esperar los resultados, guardando siempre varias formas para su propia intervención si es preciso. De esta forma, posibilita decisiones individualizadas y a la vez la adaptación del Derecho promulgado a las situaciones cambiantes sin modificar el texto de las reglas jurídicas válidas». El papel de las máximas de experiencia en el lenguaje jurídico de la determinación de los hechos es subrayado por M. Taruffo [1992, pp.204-212]. Aunque sin mención explícita a la borrosidad y a su tratamiento lógico-formal, intuiciones interesantes -junto a varias referencias bibliográficas- sobre el papel de las nociones examinadas en la toma de decisiones judiciales es suministrado por M. Taruffo [1989]. 
escala que establece su límite superior e inferior ${ }^{20}$. De las observaciones anteriores se sigue una consecuencia directa: como las formulaciones normativas son de naturaleza borrosa, también lo son los enunciados interpretativos.

La segunda fuente de borrosidad del lenguaje jurídico stricto sensu es debida a la compleja estructura de las relaciones que hay (o puede asumirse que hay) entre las diferentes normas expresadas por las formulaciones normativas cuya totalidad constituye un orden jurídico dado. En primer lugar, estamos ante una consecuencia de la borrosidad de las formulaciones normativas mismas. Como toda formulación normativa es borrosa, tenemos diferentes enunciados interpretativos los cuales -siendo a su vez borrosos indican en mayor o menor grado la corrección en relación a las normas que son expresadas por la formulación normativa. Esto es, cada enunciado interpretativo indica -con un cierto grado de corrección- cuál es la norma válida expresada por la formulación normativa. En otras palabras: en relación a los órdenes jurídicos integrados por normas legisladas, cualquier enunciado interpretativo de una formulación normativa dada conlleva un enunciado de validez respecto a la norma de la que se afirma que es expresada por la formulación normativa referida a ella ${ }^{21}$. Pero, dado que todo enunciado interpretativo de una formulación normativa es borroso, el enunciado de validez que comporta no puede ser más que borroso ${ }^{22}$. En segundo lugar, la borrosidad de los enunciados de validez está basada en la estructura de relaciones que pueden trazarse entre las normas que constituyen un orden jurídico. Para ser más preciso, la borrosidad de tales relaciones puede seguirse al menos de tres fuentes diferentes: (a) los criterios de validez de una norma jurídica pueden dar lugar a dudas sobre la legalidad de una norma dada (es el caso de una norma supuestamente inconstitucional tanto como de un

${ }^{20} \mathrm{Sin}$ referencia alguna a la borrosidad del lenguaje jurídico stricto sensu, tal rasgo de las formulaciones normativas es señalado como uno de los problemas que son desatendidos por la teoría lógico-deductiva de las decisiones judiciales e.g. por J. Wróblewski [1974, pp.42 y 45] y A. Aarnio [1977, pp.58-59].

${ }^{21}$ Una observación similar se encuentra en A. Aarnio [1981, p. 426]. «Los enunciados interpretativos y los enunciados normativos son sólo dos caras de la misma moneda», en donde «enunciado normativo» es usado con el significado de «un enunciado que dice algo acerca del contenido de una norma válida». [ibid, p.4251].

22 Tal afirmación no comporta, obviamente, sostener que una norma pueda ser más o menos válida. Simplemente señala que la cuestión de si una norma dada es válida o no, está abierta al debate y que la norma en cuestión puede conllevar diferentes enunciados borrosos de validez. A. Ross [1958, p.45] sostiene que una norma puede ser válida en mayor o menor grado. 
contrato supuestamente nulo o anulable $)^{23}$; (b) lo que habitualmente se denominan defectos de un orden jurídico -a saber: lagunas ${ }^{24}$, conflictos normativos ${ }^{25}$, y redundancia- que dan lugar, en verdad, al problema de la denominada validez de las normas (expresadas por las formulaciones normativas) que se convierten el particular sujeto de tales defectos; (c) la derogación -en su forma implícita y explícita- da lugar a problemas relacionados con la validez de las normas derogadas $^{26}$. Obviamente, las normas que se hallan en una de las anteriormente mencionadas situaciones problemáticas conllevan que los enunciados de validez sean, en mayor o menor extensión, borrosos.

(ii) Borrosidad del lenguaje jurídico de la dogmática. Por «lenguaje jurídico de la dogmática» entiendo aquel lenguaje que pretende describir lo que el orden jurídico, o simplemente alguna de sus partes, establece. En las decisiones judiciales, el lenguaje jurídico de la dogmática se usa principalmente para la justificación de las decisiones $\mathrm{y}$, a veces, puede usarse, en una cierta medida, en la formulación de la cuestión de derecho.

Sin referirse a las diferentes tesis que pueden mantenerse sobre la naturaleza, función particular y rasgos principales de la dogmática jurídica, es indudable que su lenguaje es borroso. Puede quedar para el debate la cuestión de si todos los enunciados del lenguaje dogmático son necesariamente borrosos y también hasta qué punto lo son. Pero, verdaderamente, ninguna de ambas cuestiones es preocupante, i.e.: ninguna de ellas suministra un argumento sólido para oponerse a la pretensión de borrosidad del lenguaje dogmático jurídico.

La prueba de borrosidad del lenguaje jurídico de la dogmática es aportada por aquellos de sus enunciados que comportan valoraciones de las regulaciones existentes y/o sugieren la forma en que el legislador debe mejorar o modificar determinados campos del derecho en vigor $^{27}$.

${ }^{23}$ En relación a las normas inconstitucionales, la adecuación de tal afirmación parece ser denegada por E. Bulygin [1991, p.267] y, al menos, concebida como abierta al debate por J. J. Moreso [1993].

${ }^{24}$ En un artículo que trata del aparato conceptual de la lógica borrosa en las investigaciones jurídicas, L. Philipps [1990] aborda explícitamente el caso de las lagunas.

${ }^{25}$ Los conflictos normativos son mencionados como una fuente posible de borrosidad del lenguaje jurídico stricto sensu por J. Wróblewski [1983, p.328]. Una observación similar en A. Peczenick y J. Wróblewski [1985, pp. 30-31]. Algunas observaciones sobre conflictos normativos como una fuente de incertidumbre y/o indeterminación de los órdenes jurídicos -aunque sin referencia explícita a la borrosidad- son suministradas e.g. por T. Mazzarese [1987, pp.351 -353].

${ }^{26} \mathrm{La}$ derogación es mencionada como una posible fuente de borrosidad del lenguaje jurídico stricto sensu por J. Wróblewski [1983, p.328]. Una observación similar se halla en A. Peczenick/J. Wróblewski [1985, pp.30-31].

${ }^{27}$ Ambos tipos de enunciados son mencionados en el texto como típicos de lo que he llamado lenguaje jurídico axiológico, pero como advertí en la nota 15 los diferentes tipos de lenguaje jurídico distinguidos no son mutuamente excluyentes. 
Ulterior y más importante prueba de la borrosidad del lenguaje jurídico de la dogmática puede hallarse, sin duda, en que el principal tema de la dogmática jurídica son los enunciados interpretativos y los enunciados de validez. Esto es, enunciados que -como ya se ha señaladoson borrosos. Lo que puede variar (o más precisamente: lo que puede percibirse o valorarse de forma diversa) es sólo el grado de su borrosidad.

(iii) Borrosidad del lenguaje jurídico axiológico. Por «lenguaje jurídico axiológico» me refiero al lenguaje valorativo relativo bien al derecho establecido, bien al derecho que debe ser establecido. El lenguaje jurídico axiológico ocupa un lugar destacado en la justificación de las decisiones judiciales.

No es preciso argumentar en defensa de la pretensión de que los lenguajes valorativos son naturalmente borrosos. En concreto, en relación al lenguaje jurídico axiológico se pueden señalar dos fuentes principales de borrosidad. La primera está relacionada con aquellos enunciados que suministran una valoración de las regulaciones existentes. Puesto que tales enunciados no pueden sino apoyarse en enunciados interpretativos y de validez de las regulaciones existentes.

La segunda fuente de borrosidad está relacionada con aquellos enunciados del lenguaje jurídico axiológico que sugieren las nuevas regulaciones que deben introducirse o aquellas que deben derogarse para lograr unos fines (u objetivos) dados, éticos, políticos o ideológicos. Tal cosa ocurre -no es preciso insistir en ello- porque muy raramente hay solamente una forma de lograr un fin dado, siendo fiel y coherente con un ideal determinado. En otras palabras: podemos decir que los enunciados instrumentales que señalan los medios supuestamente adecuados para lograr un fin dado son borrosos, equivalentes como son a propuestas cuyo posible éxito es una materia de grado, que puede ser de mayor o menor extensión.

(iv) Borrosidad del lenguaje jurídico de la determinación de los hechos. Por "lenguaje jurídico de la determinación de los hechos" entiendo el lenguaje usado en las decisiones judiciales para formular aquello relativo a la denominada determinación de los hechos, esto es, aquello referido a los enunciados probatorios y a la valoración de las pruebas.

No hace falta señalar que la llamada determinación de los hechos es el núcleo real de las decisiones judiciales ${ }^{28}$. Es claro que todas las decisiones judiciales dependen de la reconstrucción de los hechos en que se fundamenta el caso jurídico en cuestión. Ahora bien, tal reconstrucción es el resultado de varias relaciones complejas que existen entre diferentes elementos, los que a menudo no pueden formularse si no es en un lenguaje

${ }^{28}$ Es innecesario advertir que las observaciones siguientes sobre el lenguaje jurídico de la determinación de los hechos no se aplican a las decisiones judiciales tomadas por los Tribunales Constitucionales o e.g. por los Tribunales Supremos europeos, pues tales decisiones judiciales no se ocupan de ninguna cuestión de hecho. 
borroso $^{29}$. Para ser más preciso, lo que llamo lenguaje jurídico de la determinación de los hechos es borroso en un doble sentido, a saber (a) porque es dependiente del lenguaje jurídico, y (b) porque es dependiente del lenguaje común ${ }^{30}$.

El lenguaje jurídico de la determinación de los hechos es dependiente del lenguaje jurídico en el sentido que depende de, y está condicionado por, el lenguaje jurídico stricto sensu. Al menos cuatro argumentos fundamentan tal afirmación. En primer lugar, las diversas formas en que el actor y el demandado presentan los hechos que constituyen la base de un caso jurídico dado tanto como la forma en que el que toma la decisión judicial determine tales hechos están cargadas de juridicidad. Es así en virtud del doble vínculo que existe ente las llamadas cuestión de hecho y cuestión de derecho en toda decisión judicial ${ }^{31}$. En segundo lugar, el que toma la decisión judicial proyecta la variedad de datos relativos a un caso jurídico determinado, decidiendo qué cuenta como hechos primarios, secundarios o irrelevantes, respectivamente, en virtud de la forma, impregnada jurídicamente, en que tanto el actor como el demandado presentan tales datos. En tercer lugar, lo que el que toma la decisión judicial puede reconocer como pruebas depende de lo que, según el derecho procesal pueda contar como prueba, el derecho procesal no sólo varía en relación a sistemas jurídicos diferentes, sino también en relación a las diversas ramas de un mismo sistema jurídico. Y, en cuarto lugar, cómo el que toma la decisión debe evaluar las pruebas, depende una vez más del derecho procesal que regula el ámbito jurídico particular al que pertenece el caso en cuestión.

El lenguaje jurídico de la determinación de los hechos es dependiente del lenguaje común en el sentido de que las diversas formas de presentar los hechos en cuestión -aunque cargadas de juridicidad- están formuladas en el lenguaje común. Ahora bien, debe señalarse que en virtud de la variedad tipológica de hechos -sean primarios, secundarios o irrelevantes desde el

29 M. Taruffo, [1992, pp. 204-212] llama explícitamente la atención sobre la borrosidad del lenguaje necesario para formular lo relativo a las pruebas en la determinación de los hechos. Las observaciones de Taruffo -las que aparecen en las pocas páginas de su trabajo referidas a ello, tanto como otras también relevantes que aparecen en diversas partes de su fecundo análisis han influido y condicionado, seguramente, mi propia concepción de la borrosidad de lo que llamo lenguaje jurídico de la determinación de los hechos. Sin embargo, a continuación no seguiré tan de cerca las observaciones de Taruffo, y trataré de desarrollar las intuiciones que en su obra se encuentran mediante un modo de razonamiento parcialmente diferente y usando una terminología diferente.

${ }^{30}$ Las dos expresiones «dependiente del lenguaje jurídico» $\mathrm{y}$ «dependiente del lenguaje común» se parecen mucho y guardan estrecha relación con la expresión «dependiente del lenguaje» tal y como se usó al caracterizar la noción de normas (jurídicas). Vd., anteriormente, n. 16.

${ }^{31}$ La interacción entre la cuestión de hecho y la cuestión de derecho es un tema clásico en la literatura sobre la decisión judicial. Para algunas referencias sobre el tema y para el uso de la expresión de G. Bateson «doble vínculo» en este contexto, vd. T. Mazzarese, Cognition and Legal Decisions. Remarks on Bulygin's View [en prensa]. 
punto de vista del que toma la decisión- el lenguaje común usado para dar cuenta de ellos es ampliamente borroso ${ }^{32}$.

Si las anteriores observaciones son correctas, entonces es casi evidente que la borrosidad del lenguaje judicial (de los cuatro lenguajes jurídicos que lo integran) afecta a, y a la vez aporta medios adecuados para dar cuenta de, destacados rasgos de los componentes más importantes de las decisiones judiciales: (i) la cuestión de derecho (la premisa denominada mayor o jurídica del silogismo judicial); (ii) la cuestión de hecho (la denominada premisa menor o fáctica del silogismo judicial); (iii) la norma del caso (la llamada conclusión de un silogismo judicial); (iv) la justificación de la decisión judicial misma.

(i) Borrosidad y cuestión de derecho. El análisis de la cuestión de derecho plantea dos tipos de problemas: el primero se ocupa de las normas válidas que deben aplicarse, el segundo se ocupa de la elección del que toma la decisión acerca tanto de las formulaciones normativas a tomar en cuenta cuanto de las normas que se asume son expresadas por las formulaciones normativas realmente tomarlas en cuenta.

El primer tipo de problemas relativos a las normas válidas que han de ser aplicadas al decidir un caso jurídico dado precede de, y puede analizarse en términos de, la borrosidad del lenguaje jurídico stricto sensu y parcialmente -en tanto se haga uso de él- en términos de la borrosidad del lenguaje jurídico de la dogmática. Puesto que la cuestión de qué normas válidas han de ser aplicadas puede -en mayor o manor extensión- debatirse porque involucra enunciados interpretativos y enunciados de validez.

El segundo tipo de problemas relativos a la elección del que toma la decisión respecto a las normas válidas, las que realmente han de aplicarse, pertenece más bien a la borrosidad o naturaleza aproximada del razonamiento judicial que a la borrosidad del lenguaje en el que se formula la presentación de la cuestión de derecho.

(ii) Borrosidad y cuestión de hecho. El análisis de la cuestión de hecho -como el análisis de la cuestión de derecho- plantea dos tipos de problemas: el primero relacionado con su formulación, el segundo relacionado con la elección de una formulación determinada (o para ser más preciso: de una descripción de hecho dada) entre las diferentes posibles.

Obviamente el tipo de problemas de los que la formulación de la cuestión de hecho precede, y en los términos en los cuales puede ser analizada, radica en la borrosidad del lenguaje jurídico de la determinación de los hechos.

32 Aunque sobre bases diversas y desde diferentes perspectivas, se ha prestado atención a la variedad tipológica de hechos que aparecen en la toma de decisiones judiciales, bien como cuestiones de hecho estrictamente, bien como datos que han de probarse para establecer la cuestión de hecho de un caso dado. Vd. J. Wróblewski [1973], M. Taruffo [1992, pp. 105-136], T. Mazzarese, Cognition and Legal Decisions. Remarks on Bulygin's View [en prensa]. 
El tipo de problemas relativos a la elección de la descripción de los hechos para determinar la cuestión de hecho pertenece a la borrosidad o naturaleza aproximada del razonamiento judicial.

(iii) Borrosidad y la norma del caso. La formulación de la norma del caso (i.e. de la norma expresada por la conclusión de una decisión judicial) puede ser borrosa en mayor o menor grado. De igual forma que cualquier otra formulación normativa, i.e. que cualquier enunciado del lenguaje jurídico stricto sensu. Para ser más preciso, la norma del caso puede dar lugar a incertidumbre en relación a la interpretación de su formulación y en relación a su legalidad, esta última posibilidad deja abierto el debate sobre si la formulación de la norma cumple todos los requisitos legales procedimentales y materiales.

No hace falta insistir en que el enunciado de la norma del caso, formulado por el que toma la decisión (i.e. la norma expresada por la conclusión de una decisión judicial), participa del núcleo central de problemas, de borrosidad o naturaleza aproximada, involucrados en el razonamiento judicial.

(iv) Borrosidad y la justificación judicial. En la medida en que el que toma la decisión judicial debe justificar su decisión (a saber, debe mostrar sobre qué fundamentos descansan tanto la norma del caso como la cuestión de derecho y la cuestión de hecho), no puede sino hacer uso de enunciados borrosos que han de suministrar buenas razones para apoyar sus elecciones. De este modo, es muy probable que el que toma la decisión use amplia y extensamente los lenguajes jurídico dogmático y jurídico axiológico para mostrar por qué sus elecciones están mejor fundamentadas, o están más cercanas, o son más correctas que las otras posibles en relación a la redacción de los textos jurídicos, o a los principios generales del derecho, o a la voluntad del legislador, o a alguno de los posibles diversos valores y directrices políticos.

Parece adecuado sostener que la borrosidad o la naturaleza aproximada del razonamiento judicial afecta también a la elección del decisor relativa al modo en que se establece la justificación de una decisión judicial determinada. Simplemente en virtud de que las buenas razones que justifican una elección dada obviamente dependen -de nuevo- de una elección que es una función de un conjunto borroso de variables borrosas.

\subsubsection{DECISIONES JUDICIALES Y BORROSIDAD DEL RAZONAMIENTO JUDICIAL}

Las observaciones expresadas en 1.3.1. llevan a dos conclusiones: (a) todos los componentes constitutivos de las decisiones judiciales están formulados en un lenguaje jurídico borroso; (b) cada elemento constitutivo de la toma de decisiones judiciales no sólo es formulado por medio de una expresión lingüística borrosa en mayor o menor grado, sino que además puede comprenderse como el resultado de una pauta de razonamiento borrosa 
o aproximada. De una forma incluso más sintética las observaciones del parágrafo precedente pueden resumirse en la tesis de acuerdo a la cual todo elemento constitutivo de la decisión judicial es un resultado borroso de una pauta de razonamiento borrosa o aproximada.

Ahora bien, la segunda de las dos conclusiones antes mencionadas requiere de algunos comentarios puesto que hasta ahora ha sido simplemente considerada como una hipótesis plausible para explicar los problemas relativos a la naturaleza de las elecciones diversas de los decisores en cualquier etapa de las decisiones judiciales. El principal argumento que fundamenta la hipótesis en cuestión es que, en cualquier etapa de la decisión judicial, el decisor se encuentra realmente con datos borrosos pertenecientes a conjuntos borrosos.

Las razones para que ello sea de esta manera, en relación a la determinación de la cuestión de derecho y a la determinación de la cuestión de hecho, han sido ya adelantadas al tratar de la borrosidad del lenguaje jurídico stricto sensu y de la borrosidad del lenguaje jurídico de la determinación de los hechos, respectivamente. Un argumento ulterior en apoyo de la naturaleza borrosa o aproximada del razonamiento judicial en la determinación de la cuestión de derecho y la cuestión de hecho es suministrada por el requisito de justificarlas. Dejando de lado la naturaleza borrosa de la justificación misma, y el hecho de que no todas las regulaciones legales de las decisiones judiciales la requieren, lo que merece la pena señalar es que la justificación no sería sólo superflua sino incluso inútil si no se basara sobre la naturaleza aproximada del razonamiento judicial. Si el razonamiento judicial se siguiera de leyes lógicas necesarias e imperativas, ¿para qué sería necesario que el decisor aportara buenas razones de sus elecciones en la determinación de la cuestión de derecho y la cuestión de hecho? En realidad, si el razonamiento judicial fuera comprendido como siguiendo sólo leyes lógicas necesarias e imperativas, entonces cualquiera podría ver -sin necesidad de que el decisor aportara la justificación de sus propias elecciones- si una decisión judicial es correcta o incorrecta.

Si las observaciones anteriores son adecuadas, entonces también lo son a fortiori para el enunciado de la norma del caso (i.e. para la norma expresada por la conclusión de una decisión judicial).

Además, incluso si se toma por establecida la naturaleza borrosa o aproximada del razonamiento judicial, un problema ulterior aún nos espera, a saber, la tercera crítica dirigida a la teoría lógico-deductiva de la decisión judicial: la validez jurídica (que se aplica a las normas) tiene un comportamiento lógico ( si tiene alguno) que no es equiparable al comportamiento lógico de la verdad (que se aplica a la proposiciones de las que se ocupa la teoría silogística tradicional o la lógica clásica). De este modo, la cuestión que todavía debe contestarse es: ¿puede el reconocimiento de la naturaleza borrosa del razonamiento judicial sernos de ayuda para resolver el problema en 
cuestión? O, dicho de otra forma: ¿explican los cálculos lógicos esta crucial deficiencia de la teoría lógico-deductiva de las decisiones judiciales?

Realmente tal cuestión puede contestarse de dos formas diferentes según las dos perspectivas divergentes de contemplar la lógica borrosa. Las dos respuestas posibles están íntimamente relacionadas con las tesis del dilema formulado al inicio de este artículo.

\section{Las decisiones judiciales y la lógica del razonamiento aproximado: dos lecturas opuestas confrontadas} deducir» ${ }^{33}$.

Citando una expresión indudablemente feliz de N. MacCormick «decidir no es

Simple y clara como pueda sonar, esta expresión realmente da en el clavo: señala un problema crucial de cualquier intento de dar cuenta de las decisiones judiciales por medio de un aparato lógico-formal: i.e. el problema crucial de cualquier intento para fundamentar la racionalidad de las decisiones judiciales sobre la base firme del análisis lógico-formal. Lo cual es verdad para el aparato formal suministrado por la lógica clásica, y para el aparato formal suministrado por alguna de las llamadas lógicas no clásicas -sean una extensión o una desviación de la lógica clásica-. Esto es, no sólo la teoría tradicional del silogismo judicial sino también la lógica borrosa se enfrenta con el mismo problema.

Ahora bien, contrariamente a lo que podría pensarse, a pesar de la formulación clara y fecunda de MacCormick, la cuestión no tiene una única respuesta relativa al papel (al poder explicativo) del aparato lógico-formal en dar cuenta de la toma de decisiones judiciales. Como cuestión de hecho, esto es lo que ya ha pasado con la teoría tradicional del silogismo judicial y lo que probablemente pasará en relación a la lógica borrosa.

Hasta este momento, muy pocos trabajos han prestado atención al aparato formal de la lógica borrosa en el análisis de las decisiones judiciales, pero pueden distinguirse dos actitudes diferentes hacia su papel potencial en tal cuestión. Estas actitudes diferentes están vinculadas con las dos tesis del dilema expuestas al principio de este trabajo y sobre la base de estas tesis del dilema pueden adaptarse dos respuestas posibles y divergentes a la cuestión que MacCormick expresa sintéticamente como «decidir no es deducir».

En lo que sigue, en primer lugar (2.1.) me ocuparé de los argumentos sobre los que cada una de las tesis del dilema se fundamenta. En segundo lugar (2.2.), señalaré las dos posibles respuestas con que las tesis del dilema se enfrentan con la cuestión de decidir y deducir -en paradigmática oposición-. Al hacerlo, recordaré y esbozaré brevemente algunos de los diversos puntos de vista sobre la oposición paradigmática relativa a la teoría tradicional

${ }^{33}$ N. MacCormick [1989]. 
del silogismo judicial. Por último, (2.3.), sobre la base del análisis precedente, comentaré brevemente la posibilidad de reconsiderar la oposición racionalismo normativo/irracionalismo normativo desde una perspectiva nueva que nos permitiría librarnos de ella.

\subsection{Argumentos que fundamentan las tesis del dilema}

Como advertencia preliminar, debe notarse que la formulación del dilema en cuyo análisis me centraré surge de observaciones que son, para decirlo así, de naturaleza abductiva. Y ello porque hay pocos trabajos que se ocupen de la cuestión examinada $\mathrm{y}$, además, ninguno de tales trabajos, conocidos por mí, establece explícitamente ninguna de las dos tesis, incluso aunque alguna de ellas -al menos de forma implícita- parezca tomarse como plausible o potencialmente correcta.

La formulación ya sugerida del dilema era así:

(a) La lógica borrosa permite una reconstrucción racional de las decisiones judiciales puesto que suministra instrumentos lógicos para superar la crítica dirigida a la teoría lógico-deductiva de las decisiones judiciales. (b) La lógica borrosa no permite una reconstrucción racional de las decisiones judiciales puesto que en virtud de sus rasgos peculiares confirma más bien que supera la crítica dirigida a la teoría lógico-deductiva de las decisiones judiciales.

A pesar de la oposición, tales tesis comparten una pretensión común: el aparato formal de la lógica borrosa es más adecuado que el aparato formal del silogismo judicial tradicional para captar y detectar algunos rasgos destacados de la toma de decisiones judiciales. Sin embargo, la cuestión de si la validez jurídica tiene algún comportamiento lógico será contestada en 2.2., aunque la adecuación de tal pretensión ha sido extensamente comentada por los argumentos adelantados en 1.4.

Es innecesario decir que la adecuación de tal pretensión no suministra prueba alguna en favor de ninguna de las dos tesis. De hecho, el reconocimiento de la relevancia de la noción de borrosidad en el análisis de las decisiones judiciales no nos obliga a tomar partido en favor de ninguno de los cuernos del dilema. Esto es lo que parece ocurrir aparentemente con las observaciones conclusivas de J. Wróblewski sobre «borrosidad de los sistemas jurídicos», donde la borrosidad se contempla como una limitación, que el decisor racional deberá tener en cuenta, al intento de lograr certeza en las decisiones judiciales. En palabras de Wróblewski:

El decisor racional, al tomar en cuenta la borrosidad del sistema jurídico, es consciente de la debilidad de la ideología de la sujeción de las decisiones de aplicación del derecho y, también, de la necesidad de usar las decisiones, necesaria o intencionalmente, discrecionales de una 
busca la certeza donde no hay posibilidad de lograrla, ni en el área de la interpretación ni en la de la valide $z^{34}$.

Aunque en tal pasaje de Wróblewski, la racionalidad parece ser trasladada desde el status de una propiedad esencial de la decisión indicial al status de una actitud requerida (o, al menos, aconsejable) para el decisor, todavía está lejos de ser evidente de qué racionalidad se trata y qué pautas se supone que el decisor debe seguir para ser racional.

Una aproximación al papel de la borrosidad en relación a la posibilidad de lograr la racionalidad tan deseada de las decisiones judiciales que es, al menos parcialmente, menos prudente que la extremadamente prudente de Wróblewski, puede hallarse en M. Taruffo y L. Philipps, cuyos análisis dan argumentos que pueden considerarse como fundamento de una de las dos tesis del dilema. A pesar de que ni Taruffo ni Philipps adoptan una firme posición en favor de alguna de las dos tesis, el análisis de Taruffo descansa probablemente sobre la asunción vinculada a la primera de las tesis, mientras el de Philipps subyace probablemente a la segunda.

(i) La lógica borrosa como un medio para asegurar la racionalidad de las decisiones judiciales. Es claro que Taruffo es un defensor convencido -aunque no siempre convincente- de la racionalidad de las decisiones judiciales. La razón por la que esta pretensión de racionalidad no es siempre convincente puede parecer paradójica, ya que está fundada en un análisis -usualmente cuidadoso y rico en matices- que él mismo suministra de la mayoría de los problemas relativos al logro de los estándares de racionalidad que desea que se alcancen ${ }^{35}$. Ahora bien, la concepción de Taruffo de los cálculos borrosos se compadece con esta peculiar forma de contemplar las decisiones judiciales. Tomando por garantizado que en lo relativo a la borrosidad la mejor estrategia para asegurar la racionalidad de las decisiones judiciales es librarse de ella, y reconociendo, además, que tal estrategia no puede, a menudo, tener éxito, Taruffo sostiene que cuando tal estrategia no puede tener éxito, el aparato lógico de los cálculos borrosos puede ser un medio adecuado para asegurar la racionalidad de las decisiones judiciales.

La paradigmática oposición racionalismo/irracionalismo es la clave tanto en relación a la forma en que Taruffo formula el problema como en relación

\footnotetext{
${ }^{34}$ J. Wróbleski [1983, p. 330].

${ }^{35}$ La misma observación puede hacerse de otros convencidos defensores de la racionalidad de las decisiones judiciales. Ahora bien, vale la pena señalar que la paradoja de la pretensión de racionalidad de las decisiones judiciales, siendo conscientes de las dificultades que deben afrontarse para lograrla, es sólo aparente. Realmente tal paradoja se desvanece si se presta la debida atención al hecho de que la pretensión en cuestión está basada en argumentos más bien ideológicos que teóricos y/o explicativos. La naturaleza ideológica de tal punto de vista sobre las decisiones judiciales, a veces, no es ni tan sólo escondida u ocultada, sino establecida explícitamente y defendida. Así ocurre e.g. en M. Taruffo [1992, pp. 43-50]. Volveré más adelante sobre la naturaleza ideológica de la pretensión de racionalidad y/o a la posibilidad del análisis lógico-formal de las decisiones judiciales, vd. n. 47.
} 
a la forma de resolverlo. La formulación del problema relativo a casos en que los datos conllevan una dosis de borrosidad ineliminable es como sigue:

El problema [...] consiste en averiguar si en estos casos es también posible un procedimiento racional [...] o si la presencia de estos elementos condenan a la indeterminación y al subjetivismo cualquier razonamiento fundado en proposiciones que los contengan (subrayado mío) $)^{36}$.

La solución que se sugiere es hacer uso del aparato formal de la teoría de conjuntos borrosos, puesto que ello comporta

[la] posibilidad de construir procedimientos racionales que tengan como objeto conceptos vagos. La vaguedad, en otras palabras, no es por sí misma un factor de subjetivismo o de irracionalidad: simplemente es un carácter muy frecuente y extendido, irreducible en el lenguaje, que requiere una lógica «especial» para ser formalizado, pero que no excluye a priori toda posible racionalización. [...] El razonamiento sobre nociones vagas e imprecisas es aproximado, pero esto no implica que se trate de algo irracional o no razonable, ya que las nociones vagas pueden dar lugar a funciones lógicamente determinadas (subrayado mío) ${ }^{37}$.

Ahora bien, tal punto de vista sobre los cálculos borrosos y su aportación al análisis de las decisiones judiciales es engañoso (a) porque, al menos implícitamente, está vinculado, y puede conducir, a la falacia racionalista, i.e.: todo lo que puede ser analizado en términos del aparato lógico-formal dispone de racionalidad; y (b) porque la oposición racionalismo/irracionalismo no se aplica a lo que es el rasgo realmente peculiar de la lógica borrosa.

De hecho, en la lógica borrosa todo es borroso: los datos con los que trabaja (i.e. predicados y/o proposiciones borrosos), el conjunto de valores de verdad que se usan tanto como cualquier valor de verdad perteneciente a tal conjunto, las reglas de inferencia, los resultados que se siguen de las reglas de inferencia borrosas. Citando una clara caracterización de L. A. Zadeh:

Tal vez, la forma más simple de caracterizar la lógica borrosa sea decir que es una lógica del razonamiento aproximado. Como tal, es una lógica cuyos rasgos distintivos son (i) valores de verdad borrosos expresados en términos lingüísticos, e.g., verdadero, realmente verdadero, más $o$ menos verdadero, más bien verdadero, no verdadero, falso, ni realmente verdadero ni realmente falso; (ii) tablas de verdad imprecisas; y (iii) reglas de inferencia cuya validez es aproximada más bien que exacta. En relación a ello, la lógica borrosa difiere de forma significativa de los sistemas estándar de lógica ${ }^{38}$.

(ii) Lógica borrosa como un modelo explicativo de las decisiones judiciales. Taruffo no afirma ni niega que la lógica borrosa sea una lógica del razonamiento aproximado. De hecho, menciona esta caracterización de la lógica borrosa, a pesar de que su tratamiento de las decisiones judiciales está

${ }^{36}$ M. Taruffo [1992, pp. 206-207].

${ }^{37}$ M. Taruffo [1992, p. 208].

${ }^{38}$ L. A. Zadeh F [1975, p.4071]. 
inclinado a subrayar los procedimientos lógico formales que, como tales, podrían garantizar los estándares de racionalidad que desea lograr. En otras palabras, como ya advertí, el análisis de Taruffo comete, muy probablemente, una falacia racionalista. En el otro extremo, aunque sobre la base de una actitud muy confiada hacia la significación de esta nueva rama de la lógica formal para las investigaciones jurídicas, el punto de vista de Philipps no parece cometer, ni ser tentado por, ninguna falacia racionalista.

Ahora bien, antes de tratar de justificar esta afirmación, vale la pena señalar que la advertencia indicada al inicio de este parágrafo es particularmente adecuada en relación a las observaciones siguientes: son claramente de naturaleza abductiva, no sólo porque el trabajo de Philipps al que me referiré no menciona ninguna de las dos tesis del dilema, sino porque está centrado sobre programas de redes neuronales.

Mi pretensión según la cual Philipps no comete ninguna falacia racionalista, y que muy probablemente fundamenta la tesis de que los cálculos borrosos puedan contemplarse como modelos explicativos de las decisiones judiciales, se sigue de una clara observación. La confianza de Philipps en las aplicaciones de programas de redes neuronales precede de la apreciación de que tales aplicaciones pueden permitirnos reproducir una propiedad muy peculiar de las decisiones (judiciales), i.e.: recorrer el ámbito -más o menos amplio según el caso- de resultados diferentes, posibles y plausibles.

De este modo, por ejemplo, después de haber esbozado un caso más bien complejo relativo a un testamento, Philipps comenta el resultado proporcionado por un ordenador que trabaja con un programa de redes neuronales:

No puede decirse ni camino debería seguirse (como en programación tradicional), ni qué finalidad podría alcanzarse (como en la programación en PROLOG). Su resultado tampoco es el único claramente correcto, sino sólo una solución justificable (subrayado mío) ${ }^{39}$.

Tal afirmación puede tomarse como una prueba de que aquello en cuestión en los programas de redes neuronales -lo mismo ocurre con los cálculos borrosos- no es la pretendida racionalidad del resultado obtenido, ni su pretendida capacidad para asegurar la certeza del derecho, sino más bien ser una de las decisiones plausibles (o incluso una de las más plausibles) de las que pueden extraerse en relación al caso jurídico planteado. Más pruebas pueden obtenerse de otras afirmaciones de Philipps, como la siguiente:

Lo que proporcionan las redes es también lo que pretende la jurisprudencia: decisiones según similaridades y en razón de impresiones complejas que se derivan de una pluralidad de factores que no están muy determinados por las definiciones y que, en particular, sólo pueden configurarse de una manera aproximadamente mensurable ${ }^{40}$.

${ }^{39}$ L. Philipps [1990, p. 821].

${ }^{40}$ L. Philipps [1990, p. B22]. 
En pocas palabras, el objetivo más importante tanto de los programas de redes neuronales como de los cálculos borrosos es reproducir pautas diferentes de razonamiento y extraer decisiones de un conjunto borroso de variables borrosas. Esto es, proporcionar diferentes modelos posibles de explicación de diferentes modos posibles de razonamiento y de obtención de decisiones.

\subsection{Decidir no es deducir: Observaciones sobre la lógica borrosa}

Las dos perspectivas diferentes para considerar los cálculos borrosos que se vinculan con las tesis del dilema tratado en 2.1. nos capacitan para distinguir entre varias formas posibles y divergentes de dar respuesta al problema subyacente a cualquier intento de dar cuenta de las decisiones judiciales por medio de un aparato lógico-formal: el problema que MacCormick resume como «decidir no es deducir».

El modo en que la lógica borrosa está vinculada a las dos formas divergentes de dar respuesta al problema en cuestión puede, someramente, trazarse como sigue:

(a) Quien mantenga la tesis expresada por el primer cuerno del dilema no puede más que quedarse con una de las diferentes respuestas que ya se han dado al problema en relación con la teoría tradicional del silogismo judicial, aunque las respuestas se basen sobre diferentes aparatos lógicos de los cálculos borrosos;

(b) quien mantenga la tesis expresada por el segundo cuerno del dilema puede sostener, de manera convincente, que el problema en cuestión no se resuelve con los cálculos borrosos, por la simple razón de que estos son concebidos como posibles modelos explicativos de diferentes y posibles pautas de razonamiento que resultan en una decisión.

(i) Observaciones sobre la primera forma posible de responder al problema. En tanto los cálculos borrosos sean concebidos como medios para asegurar la racionalidad de las decisiones judiciales y de lograr la certeza del derecho, no pueden más que ser blancos de la misma crítica dirigida a la teoría tradicional del silogismo judicial: la validez de la norma del caso (i.e. la norma expresada por la conclusión de una decisión judicial) no es una cuestión de deducción lógica, sino más bien una materia de decisión factual ${ }^{41}$. Además, tenemos la razón según la cual -como en el silogismo judicial,

${ }^{41}$ H. Kelsen $\left[{ }^{1} 1965,{ }^{2} 1968\right.$, pp. 1484-1485] expone el problema de la siguiente forma: «Es posible, sin duda, que la norma general «Todos los ladrones deben ser castigados, i.e. enviados a prisión» sea válida, puesto que fue creada legislativamente, y que el enunciado «Smith es un ladrón» sea verdadero, e incluso que sea aseverado por el Tribunal competente, mientras la norma individual «Smith debe ser enviado a prisión» no sea, sin embargo, válida puesto que el Tribunal competente ha [...] fracasado al establecerla». Dicho en expresión de N. Iviacc., [1989]: «Decidir (como pretender) es un acto de voluntad y los actos de voluntad no son, en ninguno de sus sentidos, determinados por la lógica. Las decisiones se hacen, no se deducen. Decidir no es deducir». 
aunque sobre la base de un aparato lógico diferente-, concebidos como medios para asegurar la racionalidad de las decisiones judiciales y para lograr la certeza del derecho, confrontados con la crítica en cuestión necesariamente reproducirán una de las diferentes respuestas que ya se han sugerido en relación a la teoría tradicional del silogismo judicial. Esto es, para mencionar algunos ejemplos, que el aparato formal de la lógica borrosa puede ser comprendido como o bien (a) un medio para justificar la validez de la norma del caso sobre la base de la validez de las normas generales promulgadas por el legislador ${ }^{42}$, o bien (b) un medio para proporcionar una racionalización ex post de una decisión judicial una vez ya ha sido extraída ${ }^{43}$, o como una construcción racional limitada a la justificación interna de las decisiones ${ }^{44}$, o como un medio para mostrar que la norma del caso es justificable ${ }^{45}$.

(ii) Observaciones sobre la segunda forma posible de responder al problema. En la medida en que los cálculos borrosos se conciben como modelos posibles de explicación de las diversas pautas de razonamiento cuyo resultado es una decisión, la cuestión de la validez jurídica de la norma del caso no es materia de deducción lógica, sino más bien materia de decisión factual, que no hace falta explicar con detención puesto que es el objeto que los cálculos borrosos pretenden explicar. Como ya se ha dicho, los cálculos borrosos, como una lógica del razonamiento aproximado, no pretenden asegurar la certeza de los resultados obtenidos -i.e. en el caso de las decisiones judiciales el resultado obtenido es la norma válida que se sigue de las premisas establecidas- sino más bien proporcionar una herramienta lógica

${ }^{42}$ H. Kelsen $\left[{ }^{1} 1965{ }^{2} 1968\right.$, pp. 1484-1485] mantiene que el esquema silogístico puede ser un medio adecuado para representar el hecho según el cual: «la validez de la norma individual establecida par el Tribunal puede justificarse mediante la validez de la norma general establecida por el legislador».

${ }^{43}$ Esta concepción es defendida e.g. por A. Aarnio [1977, pp. 5758]: «el juicio es racionalizado ex post mediante la demostración de que un hecho jurídico determinado puede subsumirse en la regla general que debe aplicarse. La inferencia silogística, sin embargo, tiene otra, y de hecho más importante, limitación. El aspecto importante en el establecimiento de una decisión correcta no es sólo que sea obtenido de las premisas, es bastante más importante justificar las premisas usadas».

${ }^{44}$ Esta concepción es mantenida e.g. por J. Wróblewski [1974, p. 46]: «La justificación mediante la forma del silogismo es la justificación interna, en virtud de la cual no se controla la adecuación de las premisas. El papel de la justificación externa es, claramente, enorme, pero no puede explicarse mediante herramientas lógico formales».

${ }^{45}$ Esta concepción es mantenida e.g. por E. Bulygin, Cognition and Interpretation of Law [en prensa]: «Una decisión judicial es justificable de acuerdo al derecho si se sigue lógicamente de las normas jurídicas y de la descripción del caso. La justificación es una operación lógica, que es esencialmente de carácter deductivo, esto es, que puede ser reconstruida como una inferencia lógica, en la cual de un conjunto de premisas se extrae una conclusión que establece que ciertas consecuencias jurídicas son aplicables a un caso particular. Esta inferencia muestra que una decisión de aplicar estas consecuencias a este caso particular está jurídicamente justificada». 
que nos habilite para reproducir y distinguir diferentes pautas de razonamiento (judicial) que pueden llevar a resultados diversos.

\subsection{Observaciones conclusivas. La oposición racionalismo/irracionalismo normativo reconsiderada}

Alguna vez en este trabajo he pretendido que los cálculos borrosos pueden ser contemplados como un medio para escapar de la engañosa oposición entre racionalismo normativo e irracionalismo normativo, tal y como es entendida en el análisis de las decisiones judiciales. La razón más importante por la que hallo engañosa tal oposición no es que -citando a F. Waismann- «la chispa de la vida del racionalismo sea el irracionalismo» ${ }^{46}$. La razón más importante radica en que, en lo relativo a las decisiones judiciales, tal oposición se basa en argumentos de naturaleza ideológica más que explicativa ${ }^{47}$.

Ahora bien, si la atención se centra en un análisis explicativo de las decisiones judiciales, entonces los cálculos borrosos pueden ser un medio adecuado para mostrar que el razonamiento judicial es un razonamiento aproximado en virtud de algunas propiedades destacadas del lenguaje judicial. Nada hay involucrado aquí que sea necesariamente de naturaleza racional o irracional. En el mismo sentido, nada tiene que hacer la racionalidad o la irracionalidad con un ordenador que, sobre la base de un programa de redes neuronales y con un aprendizaje especial, pueda proporcionarnos -una vez se le ha dado un tema concreto para trabajar- una fuga cuyo estilo sea precisamente el estilo de J. S. Bach ${ }^{48}$.

(Trad. de José Juan Moreso)

${ }^{46}$ F. Waismann [21951, p. 143].

47 J. Wróblewski [1974, p. 37] ha señalado, en relación a la teoría tradicional del silogismo judicial, que las controversias son de naturaleza ideológica: «En el plano de la ideología de la aplicación judicial del derecho las tesis contrapuestas en la controversia relativa al papel del silogismo jurídico pueden reducirse a las dos siguientes fórmulas elementales: [1] La aplicación judicial del derecho debe seguir la tesis del silogismo jurídico, porque este es el modo de actuar valores fundamentales como la legalidad, la seguridad y la certeza; [2] la aplicación judicial del derecho no debe seguir la tesis del silogismo jurídico, porque la decisión judicial debe basarse sobre valoraciones que pretendan la adecuación óptima entre el derecho y los requerimientos de «la vida» (en el significado más amplio de este término) en la solución de un caso concreto». Ahora bien, tal caracterización de las tesis contrapuestas -de naturaleza ideológica sobre el papel del silogismo judicial parece olvidar que las dos tesis descansan sobre un presupuesto común que es muy problemático, a saber: la aplicación judicial del derecho puede «seguir la tesis del silogismo judicial». Si se está en contra de tal presupuesto, el problema real -de naturaleza preliminar- es si el silogismo judicial es un medio conceptual adecuado para dar cuenta de las decisiones judiciales. Esto es, citando a J. Wróblewski una vez más [1974, p. 38] -que «el silogismo como un esquema de razonamiento es adecuado para justificar la decisión judicial como una decisión racional, establecido que el uso de las formas lógicas del razonamiento judicial está garantizado» (subrayado mío).

${ }^{48}$ Esta aplicación específica de los programas de redes neuronales es mencionada por L. Philipps [1990. pp. 822-823]. 


\section{REFERENCIAS}

Aarnio, Aulis [1977], On Legal Reasoning. Turku, Turon Yliopsito.

-[1981], On the Truth and Validity of Interpretative Statements. Tercera parte de Aarnio, Aulis/Alexy, Robert/Peczenick, Alexsander, The Foundation of Legal Reasoning. En: «Rechtstheorie», 12, pp. 133-158, 257-279, 423-428.

Alchourrón, CarlosEduardo/Bulygin, Eugenio [1981], The Expressive Conception of Norms. En:

R. Hilpinen (ed.), New Studies in Deontic Logic. Dordrecht, D. Reidel, pp. 95-124.

Bulygin, Eugenio [1991], Algunas consideraciones acerca de los sistemas jurídicos. En: «Doxa», 9 (1991), pp. 257-279.

-Cognition and Interpretation of Law. En: Gianformaggio, Letizia/Paulson, Stanley L. (eds.), Cognition and Interpretation of Law. Torino, Giappichelli. [en prensa].

Diciotti, Enrico [1992], Vaghezza del diritto e controversie giuridiche sul significato. En: Comanducci, Paolo/Guastini, Riccardo (eds.), Analisi e diritto 1992. Ricerche di analitica. Torino, Giappichelli, pp. 97-138.

Faas, Horatio [1991], Lógica difusa. Tesis doctoral, Córdoba, Argentina.

Gizbert-Studuicki, Tomasz [1983], Vagueness. Open Texture and Law, En: «Archivium Iuridicum Cracoviense», 16, pp. 15-27.

Guastini, Riccardo [1992], Dalle fonti alle norme. Torino, Giappichelli, segunda edición.

Haack, Susan [1974], Deviant Logic. Some Philosophical Issues. Cambridge, Cambridge University Press.

- [1978], Philosophy of Logics. Cambridge, Cambridge University Press. Hart, Herbert [1961], The Concept of Law. Oxford, Oxford University Press.

Kalinowski, Georges [1965], Introduction a la logique juridique, Paris, R. Pichon \& R. Duran Auzias.

Kelsen, Hans [ ${ }^{1} 1965,{ }^{2}$ 1968], Recht und Logik. En: H. Klecatsty/R. Marcic/H. Schambeck (eds.), Die Wiener Rechsteoretische Schule. Wien, Europa Verlag, Band 2; pp. 1469-1497.

Luzzati, Claudio [1990], La vaghezza delle norme. Milano, Ginffre.

MacCormick, Neil [1989], Legal Deduction. Legal Predicates and Expert Systems. [Manuscrito].

Mazzarese, Tecla [1987], Antinomia, En: Digesto IV edizione. Sezine Civile, Torino, UTET, vol. 1., pp. 347-353.

- Cognition and Legal Decisions. Remarks on Bulygin's View. [En prensa]

Moreso, José Juan [1993], Sobre normas inconstitucionales. En: «Revista española de Derecho Constitutional», 38, pp. 81-115.

Opalek, Kazimierz [1972], Les normes. Les énoncés sur les normes et les propositions deóntiques. En: «Archives de philosophie du droit», 17 (1972), pp. 355-372.

Peczenick Aleksander/Wróblewski, Jerzy [1985], Fuzziness and Transformation: Towards Explaining Legal Reasoning. En: «Theoria», 51, pp. 24-44.

Philipps, Lothar [1990], Naheliegende Anwendungenneuronaler Netze in der Rechtswissenchaft. En «Iur PC», 11-12, pp. 820-827.

Ross, Alf [1958], On Law and Justice, London, Stevens. 
Quine, William Van Orman [1970], Philosophy of Logic. Englewood Cliffs N. J., Prentice-Hall. Taruffo, Michele [1992], La giustificazione delle decisioni fondate su standards. En: «Materiali per una storia della culture giuridica», 19 (1989), pp. 151-173.

Taruffo, Michelle [1992], La prove dei fatti giuridici. Milano, Giuffre.

Urquhart, Alasdair [1986], Many-Valued Logic. En: Dov Gabbay/Franz Guenthner (eds.), Handbook of Philosophical Logic. Volume III: Alternatives in Classical Logic. Dordrecht. D. Reidel, pp. 71-116.

Waismann, Friedrich [ $\left.{ }^{1} 1945^{2} 1951\right]$, Verifiability. En: Flew, Anthony (ed.), Essays on Logic and Language. Oxford, Basil Blackwell, pp. 117- 144.

Weinberger, Ota [1981], Normentheorie als Grundlage der Jurisprudenz und Ethik. Eine Auseinandersetzung mit Hans Kelsens Theorie der Normen, Berlin, Duncker \& Humblot.

Wright, Georg Henrik van [1951 a], Deontic Logic. En: «Mind», 60, pp. 1-15.

- [1951 b], An Essay in Modal Logic. Amsterdam, North-Holland.

- [1963], Norm and Action. A Logical Inquiry. London, Routledge \& Kegan Paul.

- [1985], Is and Ought. En: Eugenio Bulygin/Ilkka Niiniluoto (eds.), Man, Law and Modern Forms of Life. Dordrecht, D. Reidel, pp. 263-281.

Wróblewski, Jerzy [1973], Facts and Law. En: «ARSP», 59 (1973), pp. 161-178.

- [1974], Legal Syllogism and the Rationality of Judicial Decisions. En «Rechtstheorie», 5, pp. 33-46.

- [1983], Fuzziness of Legal System. En: Kangas, (ed.), Essays in Legal Theory in Honor of Kaarle Makkonen. Número monográfico de «Oikeustiede»16 (1983), pp. 311-330.

Zadeh, Lofti A. [1965], Fuzzy Sets. En: «Information and Control», 8.

- [1975], Fuzzy Logic and Approximate Reasoning. En: «Synthese», 30, pp. 407-428. 